\title{
Selection Process for Non-Releasable Birds: The First Step in Bird Welfare
}

\author{
KIT LACY \\ Cascades Raptor Center \\ Eugene, OrEGoN
}

Editor's Note: An earlier version of this article appeared in the International Association of Avian Trainers and Educators (IAATE) 2017 conference proceedings.

\begin{abstract}
The selection of ambassador animals coming out of wildlife rehabilitation facilities is an evolving process as information grows regarding long-term physical impacts of disabilities on an animal's quality of life. Ambassador animal welfare traditionally addressed nourishment, length of life, and physical safety while in human care. More facilities are now focusing on cognitive well-being, including examining if individuals are free from pain, fear, and distress as a measure of welfare. And, as more trainers are adopting choice-based training methods using the least number of aversive stimuli possible, candidate selection is the first step in the welfare process. Cascades Raptor Center has developed rigorous criteria for all birds before they are added to our team. Because many of our resident birds are wild-hatched individuals deemed non-releasable by rehabilitation facilities, it became necessary to devise a thorough assessment process. Data collected from wellness monitoring of our current bird collection coupled with over 25 years of comprehensive necropsy reports have provided information indicating that many disabilities that result in non-releasable status also preclude individuals from having a high quality of life in human care. Setting an ambassador animal up for a successful life in human care begins with appropriate, well considered selection.
\end{abstract}

Keywords: Ambassador, non-releasable, selection process, animal welfare

\section{INTRODUCTION}

Many zoological facilities in the United States and other regions rely on non-releasable birds coming out of rehabilitation facilities to fulfill their collection needs for native species, especially raptor species. While each individual animal must be assessed for their potential to be an ambassador animal separately, we believe that data from over 25 years of post mortem exams leads to generalized information regarding which bird may be a good candidate for possible assessment in the role of animal ambassador. Knowing the age and treatment history of each individual, the disability which resulted in non-releasable status, and tractability of certain species can assist in the

Kit Lacy is Education Director at Cascades Raptor Center. She graduated from the University of Oregon with a Master of Science in Animal Behavior and taught college biology for seven years before focusing on her passion for raptors. She has been a member of the IAATE Board of Directors since 2013.

first steps of assessment to determine if a bird is a good candidate for a life in human care as an animal ambassador.

Cascades Raptor Center is a 501 (c)3 non-profit located in Eugene, OR. We have a collection of nearly 50 avian ambassadors of both native and non-native species of raptors. Additionally, we have a wildlife hospital which specializes in raptor rehabilitation. Over the decades, we have had nearly all resident birds, upon death, examined by a board-certified avian pathologist. More recently, our staff veterinarian has done post mortem examinations of patients who were not releasable to better understand the healing process. These non-releasable rehabilitation birdsusing past criteria-may have once been considered suitable for placement as avian ambassadors. With our increased knowledge of the long-term health implications of certain types of injuries, most of these birds would no longer meet our criteria for consideration for the initial ambassador assessment process.

While Cascades Raptor Center has always strived to do the best by the animals in our care, we continue to drive our organization toward improving animal welfare. Through continuing education, professional experience, focused regular wellness monitoring, and a steadfast effort to improve the quality of care of the birds under our guardianship, we constantly work to raise the bar for what we consider to be best practices in the care of our avian collection. While we may have measured wellness in the past by observing a bird that was safe in their enclosure and eating well, we have broadened that outlook to include the Association of Zoos and Aquarium's wider examination of welfare to include an animal's ability to express species' appropriate cognitive behaviors and to live in a state free of pain, fear, and distress (AZA 2009 and 2015). As it is difficult to determine a wild animal's state of fear, pain, or distress (they are referred to as The Great Pretenders) (Fowler 2009), we believe that results of postmortem exams and some behavioral data we can 
observe while the animal is perimortem can give us insight into whether an individual may or may not have suffered from pain, fear, or distress during its life in human care.

Through our postmortem studies, we have found that many injuries that lead to non-releasable status also may lead to chronic, progressive and, we assume, painful conditions. Disabilities in a flighted bird may lead to compensatory movements and result in secondary debilitating conditions. Many disabilities also lead to poor balance, inability or unwillingness to perform self-care behaviors, navigation difficulties and a lack of confidence. In addition, we have increased our understanding of what it means to have birds voluntarily participate in their own care and training which may disqualify certain individuals as ambassadors as they are not tractable (willingness to build, or ease in building, a relationship)-such as most parent-raised owls.

Often, injuries that lead to non-releasable status have hidden consequences which are not detectable without subsequent radiographs and sometimes are seen only in postmortem exam. The examples we have found are numerous; a few are highlighted below.

\section{SECONDARY COMPLICATIONS FROM FRACTURED WINGS}

We have found that fractures near joints (e.g., wrist, elbow, shoulder) often lead to chronic osteoarthritis and other degenerative joint diseases. A former resident, male northern spotted owl (Strix occidentalis) suffered a fractured ulna proximal to the elbow. The rehabilitation facility where he was hospitalized immobilized the fracture site and no surgical repair was attempted since the radius was intact. During progressive wellness exams, it was noticed that his elbow continued to lose range of motion and the wing itself drooped more. Radiographs revealed degenerative joint disease (and postmortem exam showed joint fibrosis) even though the joint was never part of the initial injury. His inability to preen the feathers completely on this wing and the increased wing droop indicated discomfort and decreased ability to move the elbow joint. While this individual died of lymphosarcoma, the degenerative joint disease in the elbow did lead staff to look more closely at other birds with healed long bone fractures of the wing.

Similarly, in a rehabilitation case, a young great horned owl (Bubo virginianus) had an ulna fracture proximal to the elbow which was closed and wellaligned. After callous formation (approximately one month) the owl had self-directed physical therapy for an additional month. However, the movement therapy treatment did not improve wing extension and this bird had slightly asymmetrical flight and an inability to achieve sufficient height gain, which precluded release. Postmortem exam revealed that the distal humerus at the elbow already had signs of degenerative arthritis due to a slight misalignment of the ulna and altered articulation of the elbow joint. This arthritis was present not after years but in a very short window of time-only two months after the initial injury.

A second rehabilitation case study of a young redshouldered hawk (Buteo lineatus) with a compound, mid-shaft fracture of the humerus showed even more secondary issues post recovery. The surgically repaired fracture was immobilized for a month. After two months of passive movement physical therapy-after callus formation-the bird was able to fly; however, wing extension was inadequate for release. Upon further examination postmortem, it was discovered that the break had not calcified. Cartilage was present between bone ends resulting in a non-union. A tendon had adhered to the humerus and both the shoulder and elbow had altered joint articulation. Inflammation was already present, especially in the shoulder, which would indicate the development of arthritis in only three months in human care.

Finally, a young turkey vulture (Cathartes aura) with an old, badly healed, fractured humerus underwent surgical repair and bone pinning by a board-certified orthopedic veterinarian. Complications resulted post-surgery and the bird was humanely euthanized. While we knew the surgical repair of the wing was not successful, we did not appreciate the severity of the complications, due to this bird's calm temperament. Postmortem revealed that bone marrow was exposed and periostitis (bone lining inflammation) had developed in the three months post-surgery. Both conditions, it could be assumed, would lead to an extreme amount of pain. However, this bird was eating and appeared 'comfortable' while in care. This could have been the result of a 'preservation reflex.' Wildlife is conditioned to hide physical pain to the very end in order to survive. We would never consider a bird eating as an indication of comfort-only an indication of hunger. An individual with this level of disability would certainly not be "free of pain, fear or distress" while in human care if he had been placed (NIH 2000). In these examples of fractures, we have found the following:

- Fractures near joints will lead to probable osteoarthritis and/or other degenerative joint disease; 
- Fractures that result in mis-alignment and altered articulation of joints may also lead to osteoarthritis and/or other degenerative joint disease;

- Long bone fractures of the wing may also lead to other painful conditions such as bone inflammation or exposed bone marrow.

As stated in the Manual of Ornithology: Avian Structure and Function "Nothing about the construction of the bird's wing is arbitrary" (Proctor and Lynch 1998). The bones of the avian wing have been fused and greatly reduced to a point that every bone in the wing serves a complex function, not only for flight but for overall mobility. Altering or removing any part of the wing, therefore, has the potential for serious consequences to the individual.

\section{SELF-CARE}

Self-care (e.g., preening, bathing, maintaining good feather condition) is essential for a bird in human care. We believe it can also be a measure of confidence and their overall level of physical comfort. Disabilities can often lead to poor balance and reduced mobility, and therefore an inability to perform self-care. Feathers may remain unpreened near or sometimes distal to joints that are injured, blood feathers may break, and other feathers may become damaged due to either the inability to reach the feathers or pain while doing so. While we cannot say for certain that it would be distressing for a bird to be unable (or unwilling) to perform self-care, we do know that feathers are an essential physical aspect of birds and responsible for flight, thermoregulation, camouflage, and breeding success. Activity budgets of wild birds estimate that up to 10 percent of the day may be spent in preening. Additionally, feathers that are 'in-blood' are very sensitive with nerves and blood vessels attached; breaking them is painful and potentially fatal.

Injuries near joints can prevent proper self-care. A former resident adult female merlin (Falco columbarius) with healed fractures of the radius and ulna resulting in a contracted right carpal joint was unable to preen her primaries and some covert feathers on the restricted wing. An adult bald eagle (Haliaeetus leucocephalus) was presented for care in our wildlife hospital with a traumatic amputation of the distal 40 percent of the second phalanges of one wing. While this individual was not particularly fractious, even in a low human-contact setting, he was unable to successfully grow feathers and continued to damage and break nearly all his primary flight feathers as they grew. While a more drastic amputation of the wing tip may have prevented the damage and breaking of feathers in human care, our team felt that quality of life would be so drastically decreased due to amputation that euthanasia was the most humane option.

Shoulder injuries can lead to long-term inability to care for feathers as well. A former resident golden eagle (Aquila chrysaetos) with a dislocated shoulder was unable to preen feathers on his increasingly immobile wing. As he aged, the occurrence of broken blood feathers increased as his navigation ability decreasedsometimes leading to falls despite rearrangement of enclosure ramps, perching, and platforms to accommodate his decreased mobility. Due to this inability to care for himself (and other age-related health concerns), it was determined that the humane course of action would be euthanasia in this case even though he continued to eat well, build a nest, and interact with trainers.

Self-care is not just about feathers. Iatrogenic injuries (those that occur while an animal is in care) may also occur. In the case of birds who have any part of their wing amputated, the site of amputation is at great risk for injury as it is no longer protected with the rest of the wing, and the bird's ability to navigate its space is compromised. Therefore, any wing amputation may result in poor balance and, sometimes, falls from perching. An adult, resident bald eagle who lost the tip of his wing often falls when attempting to avoid a perceived dangerous situation and injures the amputation site. While not life-threatening, these injuries are certainly painful.

\section{COMPENSATORY MOVEMENT DISABILITY}

Disabilities that lead to non-releasable status often decrease the bird's ability to ambulate freely. Injuries that alter or decrease the ability of the individual to move freely can also lead to compensatory movement disability. Additionally, the lack of ability to evade any perceived threats may decrease an individual's confidence as they are no longer able to adequately move about their environment. In the case of a former resident bald eagle, a traumatic injury to the elbow lead to degenerative joint disease and non-releasable status. As she aged, we noticed that her ability to move about her exhibit space also decreased. It was as if she no longer had the use of the wing from the shoulder. This individual had come to our facility as a 'glove trained' bird; however, when she arrived it was apparent that she was a case of learned helplessness. Her confidence on display to the public and her willingness to work with trainers continued to decline, even as we gave her more choices. Upon taking radiographs, we found that her wrist (in addition to the 
original elbow injury) had signs of severe degenerative arthritis. This arthritis was possibly due to compensatory movements resulting from her original injury. Her shoulder also had limited range of motion and she was unable to open the wing fully. To our knowledge, neither the wrist nor shoulder conditions were the result of her initial injury.

A second case was a former resident burrowing owl (Athene cunicularia) with a fractured and healed right ulna which bridged to the radius changing the joint articulation and resulting in immobility of the wing. As she aged, we noticed a limited use of the left leg and sometimes a limp. While we could appreciate some swelling in the hock joint on physical exam and radiographs, we did not discover until the post mortem that her patella had fused to both the femur and tibia due to cartilaginous proliferation. This fusion resulted from compensatory movements she was using to navigate her exhibit enclosure due to her disabled wing.

\section{TEMPERAMENT}

Finally, we have found that with our facility's increased understanding of what it means to have birds voluntarily participate in their own wellness and training sessions, some individuals may not be appropriate candidates for ambassadors (AZA 2015; Martin 2016). Because of past history and their unwillingness to participate voluntarily, despite extended relationship-building and training attempts, these birds do not develop the confidence for a high quality of life in human care. In all rehabilitation patient cases, the decision to treat is based on that individual's release potential not their potential to be an educator. Our rehabilitation staff believes that a bird who is terrified, in extreme pain, and in a small enclosure cannot be evaluated for their tractability. We have found that it is very difficult to assess a bird's suitability for the job of ambassador before treatment is complete. Pain, shock, and terror often mask the bird's tractabilityleading to a sense of false potential for a life in education. Once a bird has progressed through treatment, severe flight disability may lead to learned helplessness as well.

Additionally, two cases of post mortem results of two parent-raised owls showed signs of chronic hypertension which may have been the result of chronic stress of being in human care. These birds had chronic arteriosclerosis lesions which could be a result of their long lives but may have been aggravated by high levels of stress as suggested by the pathologist. Both birds would sit calmly in their exhibit enclosures and did not demonstrate outward signs of stress, however, neither of these owls would voluntarily participate in training sessions with our handlers.

Considering these findings over the years, we believe the following conditions in non-releasable birds result in disqualification as candidates for education ambassadors.

- Injuries near joints

- Most amputations

- Injuries that result in undue stress on joints by changing joint articulation

- Most adult or parent-raised owls

- Disabilities that decrease confidence

- Any other condition that may result in or develop into on-going pain, distress and/or fear

While the term 'confidence' may be nebulous when referring to wild animals, we believe that there are few criteria that we can use to help determine the suitability for life in human care.

- If the individual can live comfortably and safely in a free-lofted situation and willingly participates with trainers, they may be confident. While tethering is an essential part of working with raptors (see IAATE Tethering Position Statement, IAATE 2015), we do not believe it should be the primary training tool used during initial training of a raptor.

- The bird positively anticipates training sessions while at a healthy weight and fit body condition.

- The bird does not engage in avoidance behaviors.

- If the bird is willing to share personal space with the trainer and receives reinforcement for behaviors.

Cascades Raptor Center staff asks the following questions before adding a bird to our ambassador collection.

- Is the bird able to move around the enclosure freely and safely? Can the bird use the highest possible perching, well out of the reach of any trainer?

- Is the bird able and willing to make choices in an empowerment-based training environment?

- Is the bird able and willing to perform normal self-care behaviors?

- Does the bird have an intact sense of confidence or empowerment (a sense of control)?

Once a bird has (a) progressed through rehabilitation and (b) the disability is determined to (most likely) not cause any on-going pain, distress or fear, and, (c) the individual meets our additional criteria for candidacy, our staff begins our ambassador assessment 
and initial training program. We do not refer to this as "manning" because we feel that term refers to grabbing a bird, putting equipment on it and then holding them on the glove until the animal discovers it cannot get away. Our assessment process goal is to free-loft the bird using the least aversive stimuli possible, allowing the bird the choice of interacting with trainers for positive reinforcement (Martin 2016).

Although these criteria may seem to offer bleak prospects for the vast majority of wild birds coming through rehabilitation and deemed non-releasable, we feel that humane euthanasia is ethically more appropriate than a life of potential pain, discomfort, and fear under human care. If it is felt that a bird-despite demonstrating some of the potential problems listed above-should be considered for education, facilities should have a rigorous wellness program in place to continually monitor each bird for signs of pain, fear, or distress and be ready to make the always difficult but often necessary decision to euthanize. Staff at Cascades Raptor Center are also interested in researching long term pain management protocols that might alleviate pain and prolong a life in comfort without causing other problems, such as kidney compromise.

Each bird is an individual and must be assessed accordingly. Our facility's commitment to professionally trained staff to oversee the training, handling and wellness of our residents is paramount. We will also continue to improve our trust- and choice-based training techniques. And, we will always evaluate our ability to provide care before considering the addition of any new avian team member through a collection plan.

As with any process, we are learning and growing as we proceed. We are not perfect, and we hope that data we have acquired and lessons learned will help us-as well as the community of like organizations-to continue to provide the best possible care to our collections today and our collections in the future.

\section{ACKNOWLEDGEMENTS}

There are many people to thank for their continued work at Cascades Raptor Center and they are all part of the team that made this paper possible. Thank you to Louise Shimmel for having a dream, making it a reality and sharing it with me. Thank you to Laurin Huse for being there for all the discussion on how best we can care for the birds. Dr Uli Streicher, Dr Madeline Rae, and Dr. Devin Newman made this paper possible for the care they help us offer the birds, but also the invaluable postmortem information they provide to our facility.

\section{LITERATURE CITED}

Association of Zoos and Aquariums. 2015. Ambassador Animal Policy. Accessed Jan. 2017. Available from: <https://www.aza.org/ aza-ambassador-animal-policy>.

Association of Zoos and Aquariums. 2009. Animal Welfare Committee. Accessed January 2017. Available from: <https://www.aza.org/ animal_welfare_committee>.

Fowler, A. Recognition of Pain in Wildlife. 2009. Accessed January 2017. Available from: <http://www.fourthcrossingwildlife.com/ RecognitionOfPainInWildlife-AnneFowler.pdf>

International Association of Avian Trainers and Educators (IAATE). 2015. Position Statement: Tethering and the Use of Jesses. International Association of Avian Trainers and Educators. Accessed October 2018. Available from: <https:// iaate.org/images/Position_Statement_-_ Tethering_and_the_Use_of_Jesses_Revised.pdf >

Martin, S. 2016. Improving Animal Welfare Through Training. Proceedings of IAATE 2016 Conference. International Association of Avian Trainers and Educators..

National Institutes of Health. 2000. Definition of Pain and Distress and Reporting Requirements for Laboratory Animals: Proceedings of the Workshop National Research Council (US) Committee on Regulatory Issues in Animal Care and Use. National Academies Press: Washington, DC.

Proctor, N. S. and P. J. Lynch. 1998. Manual of Ornithology: Avian Structure and Function. Yale University Press: New Haven, CT.

\section{RECOMMENDED READING}

Grandin, T. and C. Johnson. 2005. Animals in Translation: Using the Mysteries of Autism to Decode Animal Behavior. Harcourt: New York, NY. (N.R日 